www.nature.com/clinicalpractice/neph

\title{
Acute kidney injury/acute renal failure: standardizing nomenclature, definitions and staging
}

\author{
Suzanne J Farley
}

More than 30 different definitions of 'acute kidney injury' (AKI) appear in the published literature. The use of different definitions in different studies makes it virtually impossible to analyze outcomes data in any meaningful way. If the efficacies of treatment strategies cannot be reliably compared, how are physicians to decide which approach to the management of this disorder is best? Having an evidence-based answer to this question is crucial if the dire mortality rate currently associated with AKI is to be improved.

To this end, the Acute Kidney Injury Network (AKIN) has been formed. Representing nephrology and critical care societies, as well as the Acute Dialysis Quality Initiative, AKIN's remit is to "facilitate international, interdisciplinary and intersociety collaboration that will ensure progress is made in the field of AKI." In this issue of Nature Clinical Practice Nephrology (see page 439) you can read a summary of the first AKIN meeting. The aim of the first conference was to create a standard definition of, and staging system for, AKI. Modeled on RIFLE criteria, and based on the relatively simpleto-measure variables of serum creatinine and urine output, these new standards should be amenable to widespread implementation once validated.

Of course, it could be argued that serum creatinine level and urine output are not the most accurate indicators of renal function. For example, even very small changes in serum creatinine concentration can be associated with adverse outcomes, and detecting early declines in glomerular filtration rate is difficult. Clinical availability of more-accurate markers of renal injury would enhance detection of AKI and timely initiation of intervention. Candidate biomarkers in both plasma and urine, such as neutrophil gelatinase-associated lipocalin, cystatin C and kidney injury molecule-1, are being intensively investigated. Until a highly sensitive and specific panel of biomarkers that can be easily used in practice has been validated,

\section{...naming \\ an entity \\ accurately \\ is the first \\ step towards \\ generating \\ a correct \\ definition}

SJ Farley is Editor of Nature Clinical Practice Nephrology.

Competing interests

The author declared no competing interests.

www.nature.com/clinicalpractice doi:10.1038/ncpneph0563 however, standardizing current definitions and classification of AKI is the optimum approach.

The need to standardize AKI definitions is perhaps most pressing in pediatric populations. On page 408 we highlight the pediatric RIFLE criteria. Modified from the adult definitions by Stuart Goldstein and colleagues, this new staging system was prospectively applied to 150 critically ill children. If used by many clinicians, the pRIFLE criteria could help to optimize the evaluation and management of pediatric AKI.

Dialysis is one of the cornerstones of AKI treatment. Unfortunately, as with many interventions, adapting dialysis for effective pediatric therapy is problematic. 'Downsizing' standard dialysis equipment for use in neonates of low birth weight is particularly challenging. A Research Highlight on page 408 summarizes a recent advance reported in Medical Engineering \& Physics. The new automated hemodialysis system does not require continuous vascular access, needs only a very small volume of blood for priming (obviating the need for donated blood), and affords direct control of clearance and ultrafiltration rates. At the time of publication, the new system had been used to successfully treat seven premature babies with AKI or metabolic disorders.

You might have noted in this article that the term 'AKI' has been used in favor of 'ARF' (for 'acute renal failure'). This issue of semantics might seem trivial relative to the clinical issues that need to be resolved; however, naming an entity accurately is the first step towards generating a correct definition. The term 'AKI' has been favored more recently on the basis of the fact that the condition does not always result in kidney failure, as would be indicated by 'ARF'. A quick search of titles and abstracts listed in PubMed shows that 'AKI' - first used in 1990-has now been used 116 times, the vast majority in the past 2 years. There's still a long way to go, however, before the number of articles in which this term is used exceeds that of 'ARF'-almost 15,000. 